\title{
Conditions for the use of simulators that implement the features of artificial intelligence systems when studying at a transport university
}

\author{
M. V. Karelina*, and S.P. Vakulenko \\ Russian University of Transport( MIIT), 9 Obraztsova str., 9, 127994, Moscow, Russia
}

\begin{abstract}
The article presents the main conditions for the use of simulators, which are proposed to be used both for the simulators themselves, which implement the features of artificial intelligence systems, and for the organization of work with simulators in the educational process. Training of students with the use of simulators should form practical skills of the future specialty in accordance with the types of professional activity and qualification characteristics. The conditions for the use of simulators are proposed to include: the implementation of the activity approach; information security of software products; leveling of possible negative consequences for the health of the student, etc. Each of the presented conditions implies the need to ensure the formed parameters and the order of their evaluation. It is concluded that the above conditions for the use of simulators will allow for the development of new forms of professional training and appropriate scientific and methodological tools, and the need to actively introduce new high-tech simulators into the educational process of transport universities, including those that implement the capabilities of virtual/ augmented reality technologies.
\end{abstract}

\section{Introduction}

One of the priority tasks of the higher technical education system is to prepare specialists for professional activities. Currently, there is an active expansion of the use of modern teaching tools in the educational process, operating on the basis of digital technologies, implementing the features of artificial intelligence systems and forming high-quality training of railway transport specialists. The improvement of scientific and technical achievements in the field of high-tech equipment and software has led to the development of technologies that provide modeling, simulation, simulation, visualization, in which three-dimensional modeling is actively used in the training of future specialists, allowing you to simulate existing and abstract models in detail and visualize them in the environment of real-world objects. Changes in education related to the need to use digital technologies [1] determine the feasibility of developing new software and hardware tools and teaching methods, including the development of requirements and conditions for the use of high-tech simulators and the implementation of psychological, pedagogical, educational and methodological support in

\footnotetext{
* Corresponding author: mv_karelina@mail.ru
} 
the conditions of ensuring the safety of students when using high-tech simulators with virtual and augmented reality technologies.

Materials and Methods. The research is carried out on the basis of theoretical methods.

Results and Discussion. Under the concept of high-tech simulators, we will represent complex software-adapted systems that include elements of simulation and simulation of the objects being studied, physical or screen models, the implementation of which provides simultaneous perception of objects, processes, scenes of real reality and virtual reality by the user [2].

The system of training students of transport universities with the use of simulators should provide the tasks of forming practical skills of the future specialty in accordance with the types of professional activity and qualification characteristics. The technical design of the simulators used in the training system, as well as their software, must exactly correspond to the new technical means, have the same controls and assume the possibility of modernization, depending on the changes made to the design of the technical means.

This leads to the need to develop a set of conditions, both for the simulators themselves, which implement the features of artificial intelligence systems, and for the organization of work with simulators in the educational process [3].

The conditions for the use of simulators in the educational process that implement the features of artificial intelligence systems can be structurally divided into:

- conditions for the organization of work on simulators;

- conditions for the implementation of the activity approach in training on simulators;

- conditions of information security of software products during training on simulators;

- conditions for leveling possible negative consequences for the health of the student when using simulators;

- content and pedagogical conditions for the use of simulators in the educational process.

Conditions for the organization of work on simulators that implement the capabilities of artificial intelligence are provided:

-the duration of stay in the visual environment, in accordance with the type of simulator used;

- minimization of operations in preparation for work;

- reliability and safety of the simulator operation;

- checking the visualization of the simulator systems;

- the level of sound and vibration in accordance with the technical parameters of the simulator.

\subsection{Duration of stay in the visual environment}

Determine the permissible working time on the simulator should take into account the type of simulator used, the types of professional activity and the qualification characteristics of the future professional activity of the specialist. Choose a specific type of simulator should take into account the methodological methods of training used and the possibilities of technological and technical implementation of simulators. Taking into account the methodological method of training and in accordance with the type of future professional activity of the specialist, the permissible working time on the simulator must be determined for training: on simulators of future employees of the locomotive economy; future employees of the dispatching apparatus and personnel of the transportation service; future employees of the track infrastructure; future employees of the automation and telemechanics economy; future employees of the passenger economy; future employees of the freight economy. 


\subsection{Minimization of operations in preparation for work}

To make sure that the minimum number of operations can be used to prepare for work, it is necessary, taking into account the compliance of the operations performed with the accepted technology of working on the simulator, by acting on various switches and control buttons, making sure that there is the least amount of time required to turn them on or move them, that there are no inconveniences and that they work.

\subsection{Reliability and safety of the simulator}

To realize the possibility of reliability and safety of training on the simulator, it is necessary: - make sure visually that the lighting of the devices and equipment is working properly and that they can be adjusted with the help of switches;

- make sure that the switches and control buttons that are responsible for adjusting the time for detecting damage and non-standard situations, making decisions and managing activities are working properly. It is possible to determine this time with a stopwatch by determining the response time to pressing the control buttons;

- make sure that the temperature mode can be controlled using the switches;

- make sure that the communication means between the students and the instructor is working properly and can be adjusted by pressing the various switches and control buttons and receiving a response;

- make sure that the dynamic chair used is working properly, that the backrest and armrests can be adjusted, which must be positioned at a height at which the feet will be firmly on the floor;

- verify the operability of the opening structural elements (doors) and the possibility of adjusting the structural elements by visual inspection;

- make sure that the tools designed to provide information about the progress of the educational process are working properly, that the actions of the trainees are proportionate when managing the simulated system and that they can be adjusted by running any 2-3 programs, and visually make sure that they are working properly when running;

- make sure that the buttons and switches are not pushed without permission, by influencing them and receiving a response;

- make sure that the system can be quickly and easily restored to its original position, by acting on the switches, receiving a response and checking the health.

Checking the visualization of the simulator systems.

Verify visually the stability of colors used on the screen (for example, the red color is a problem or error; yellow-warning, green-approval, etc.);

Verify visually that the video quality and sharp, clear, without the phenomenon of compression and impressions of the "jitter" of the camera, the frame rate is not more than 60 frames per second, minimum resolution $1280 \times 720$ pixels;

Make sure visually that there are no "freezes" in computer graphics, image resolution depending on the screen size( dpi), color depth High Color or True Color, color model CMYK, HSB;

Visually verify the background brightness measured in the dark, cd / $\mathrm{m} 2$ (Min. value-35, max value-120);

Make sure that the screen is illuminated visually, lux (Min. value-100, max. value-300);

Visually verify the size of the corner mark by determining it with a caliper, mm (Min. value-16, max. value-80). 


\subsection{Sound and vibration level}

Make sure that you can adjust the volume level and adjust them using the switches;

Make sure audibly with careful listening that there is no noise that appears when technical systems fail, side interference, sharp, clear or too high-pitched sounds;

Make sure audibly when listening to the absence of sound effects caused by the operation of the devices, obvious, hard and exaggeratedly loud sounds of the devices;

Make sure that there is no dynamic impact, "uncharacteristic" shocks and impacts, the delay or deceleration time of the simulated acceleration data on the simulator, which should not exceed 0.1-0.2 seconds. In terms of stability to the influence of external climatic factors, the simulator must comply with Group 1 of GOST 21552-84.

The conditions for the implementation of the activity approach in training on simulators that implement the features of artificial intelligence systems are as follows:

- to form the content composition of the competencies of students of the transport university in the field of: practical training on transport simulators that implement the features of artificial intelligence systems; skills in the operation of transport systems, in accordance with the level of training of students, which can be directly used in a professional situation $[4,5]$;

- implement situations of professional activity (operation of existing transport control systems, management of transport and logistics systems, transport flows, etc.) that require analysis and decision-making based on theoretical information in the field of using the features of artificial intelligence systems and integrating elements of artificial intelligence and the capabilities of robotic devices in transport systems; [6,7];

- implement the capabilities of the simulator for memorizing, evaluating and analyzing the actions of the student while practicing practical skills;

- select the complete set of the training simulator adequately to the training tasks, in accordance with the types of professional activity and qualification characteristics;

- implement organizational forms, methods and means of teaching adequately set educational goals and personal potential of the student [8].

The conditions of information security of the applied software products associated with the use of simulators in the educational process that implement the features of artificial intelligence systems are as follows:

- organize the protection of the information contained in the simulator software that implements the capabilities of artificial intelligence systems $[9,10]$;

- organize the provision of accessibility and continuity of information (materials) contained in the simulator software that implements the features of artificial intelligence systems in an undistorted form [12];

- to carry out educational activities that neutralize the risks associated with the use of simulators in the educational process that implement the features of artificial intelligence systems $[13,14]$;

- implement a set of measures to control and manage changes in information in simulators that implement the features of artificial intelligence systems;

- to provide equipment and means of information protection in simulators that implement the features of artificial intelligence systems [15];

- provide means of protecting the information available in simulators that implement the capabilities of artificial intelligence systems: from unauthorized exposure; from loss (leakage); from accidental and malicious exposure.

The conditions for leveling possible negative consequences for the health of the student when using simulators that implement the features of artificial intelligence systems are as follows:

- observe the rules of not allowing distortions in the content, methodological and methodological approaches and violations of the principles of pedagogical expediency [16]; 
- observe the sanitary and hygienic and medical requirements in the field of health preservation (vision, musculoskeletal system, neuropsychiatric sphere, etc.) when working on simulators in conditions of simultaneous stay of the student for a long time sitting, in a stationary position [17];

- to provide psychological comfort to the student when interacting with the simulator: to warn about the inadequacy of his perception of reality after working on the simulator; to warn about an unreasonably high level of" trust " in the automated system more than to himself, which can be dangerous due to a decrease in conscious control.

The content and pedagogical conditions for the use of simulators in the educational process that implement the features of artificial intelligence systems are as follows:

- ensuring interactivity, the possibility of modeling objects of professional activity, increasing the volume and types of information provided simultaneously, when organizing the interaction of the student with the simulator (technical means) and the teacher $[18,19]$;

- providing visualization and modeling of objects in accordance with the types of professional activity and qualification characteristics, realizing the capabilities of the simulated objects of professional activity and increasing the number and types of information provided simultaneously [20,21];

- providing simulation of interaction with objects, participation in virtual processes and training situations," presence " of the student in virtual processes, and situations when working on the simulator [22];

- adaptation of digital content to the capabilities of the student, including when monitoring the results of mastering the educational material.

- ensuring the individualization of the learning process by the time of the actual work of the student with the simulator to form his personal trajectory and record the results of learning development;

\section{Conclusions}

The complex of the listed conditions for the use of simulators that implement the capabilities of artificial intelligence will allow for the development of new forms of professional training and appropriate scientific and methodological tools aimed at transforming the methods of professional training and mastering new competencies by students of transport universities and intensifying the learning process. At the same time, we can talk about the need to actively introduce new high-tech simulators into the educational process of transport universities, including those that implement the capabilities of virtual/ augmented reality technologies, allowing you to create various standard and non-standard situations, in conditions of preserving the health and protecting the information security of the students ' personality.

\section{References}

1. I. V. Robert, Theory and methodology of informatization of education (psychological, pedagogical and technological aspects), 398 (2014)

2. M. V. Karelina, Pedagogical informatics, 2, 48 (2019)

3. M. V. Karelina, Humanitarian Scientific Bulletin, 2, 42 (2020)

4. N. F. Talyzina, Management of the process of knowledge assimilation (psychological foundations), 344 (1984)

5. N. F. Talyzina, Workshop on educational psychology, 189 (2002)

6. E. George, D. George, Artificial intelligence: what you should know about the coming era of intelligent machines, 348 (2019) 
7. Stewart Russell, Peter Norvig, Artificial Intelligence: A Modern Approach (AIMA), 1424 (2007)

8. S. P. Vakulenko, P. A. Egorov, E. V. Kopylova, E. B. Kopylova, Applicant, 2(8) (2010)

9. Yu. V. Bogatyreva, O. A. Kozlov, V. P. Polyakov, A. N. Privalov, Theoretical and practical aspects of psychology and pedagogy, 27 (2017)

10. I. S. Klimenko, Information security and information protection: models and methods of management, 180 (2020)

11. Yu. N. Klimenko, Information security standards. Protection and processing of confidential documents, 223 (2020)

12. V. P. Polyakov, Aspects of information security in information training, 135 (2016)

13. S. A. Beshenkov, Ya. A. Vahramenko, V. A. Kastornova, O. A. Kozlov, E. V. Mindzaeva, et al, Development of informatization of education in school and pedagogical university in the conditions of ensuring information security of the individual, 107 (2018)

14. V. P. Polyakov, Information security of the person of subjects of educational process in modern societies, 323 (2020)

15. E. V. Vostretsova, Basics of information security: a textbook for University students, 204 (2019)

16. Theory and methodology of informatization of education (psychological, pedagogical and technological aspects), 398 (2014)

17. I. Sh. Mukhamedzyanov, Health-saving education: essence and technology, 218 (2011)

18. O. P. Belova, M. V. Kotkina, A. A. Kaznin, A.V. Ananyin, 3D-modeling and augmented reality, 90 (2018)

19. Augmented, Virtual Reality to hit $\$ 150$ billion disrupting mobile by 2020 , http: //www.digi-capital.com /

20. H. Papagianis, Augmented reality, Everything you wanted to know about the Technology of the future, Helen Papagianis, 288 (2019)

21. M. Broussard, Artificial intelligence: the Limits of the Possible, 361 (2020)

22. I. V. Robert, Directions of development of informatization of domestic education in the period of digital information technologies, 23, 1 (2020) 